\title{
Comment encourager et promouvoir l'innovation en chirurgie?
}

Les opinions exprimées dans cet éditorial sont celles de l'auteur et ne représentent pas nécessairement celles de l'Association médicale canadienne ou ses filiales.

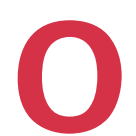
$\mathrm{n}$ ne s'entend pas sur la définition exacte du mot innovation. Certains chirurgiens estiment qu'il s'agit d'une idée, d'une méthode ou d'un instrument inédits. D'autres sont plus nuancés et parlent de la création, de la mise au point et de l'application d'un produit, d'un processus ou d'un service nouveaux. Cette dernière définition est particulièrement intéressante si on la combine à un objectif d'amélioration de l'efficience, de l'efficacité et/ou à un avantage concurrentiel. Selon une définition encore plus audacieuse, l'innovation est une « rupture technologique » qui modifie le paysage et découle en général des progrès scientifiques et technologiques ${ }^{1}$.

Peu importe le domaine (chirurgical ou autre), une « invention » n'est que le premier élément d'une réelle innovation. Le processus entier est peut-être le mieux décrit par la courbe dite du « 0 à $100 »^{2}$. L'étape initiale, de « 0 à 1 », coïncide avec l'avènement d'une rupture technologique qui a le potentiel de modifier un domaine donné. L'étape subséquente, de « 1 à 100 », repose sur une succession de progrès consécutifs (p. ex., globalisation et imitation) attachés à cette rupture technologique au fil du temps ${ }^{2}$. Même si en général, l'étape de 0 à 1 représente moins de $0,1 \%$ du processus, les deux étapes du parcours sont cruciales pour réaliser le cycle complet de la rupture technologique et en assurer la pérennité. Le meilleur exemple du parcours de 0 à 1 et à 100 , et donc, l'illustration par excellence de l'interconnexion entre les premiers balbutiements d'une invention et sa maturation, est celui de l'automobile. Depuis ses humbles débuts en 1896 en tant que véritable rupture technologique 0 à 1 (c.àd., le remplacement du cheval pour la traction des véhicules), nous avons été témoins des multiples progrès de l'ingénierie des véhicules moteurs et ces progrès nous amènent au bord d'un autre gouffre, ou d'une autre rupture technologique potentielle de 0 à 1 dans le domaine des transports. En effet, la création de véhicules autonomes sans chauffeur, provoquera d'importants changements dans notre façon d'occuper le territoire, de nous déplacer, de construire des routes et des infrastructures; elle modifiera notre façon de prodiguer des soins en cas d'accident/traumatisme/blessure et transformera même la configuration de nos environnements de travail (p. ex., espaces de stationnement).
Les chirurgiens sont particulièrement bien placés pour reconnaître ces différents parcours de l'innovation et pour y jouer un rôle actif. Même si les aspects fondamentaux de l'innovation au sein du système de santé vont de pair avec ceux d'autres domaines (p. ex., économie, processus internes, nouvelles offres et modèles de prestation), les occasions d'innovation abondent autour de nous sur une base quotidienne. Pensons entre autres à la télémédecine, à la médecine personnalisée, aux cliniques médicales intégrées à des commerces, à l'intégration de l'intelligence artificielle et, bien sûr, à la création de dispositifs dans des espaces chirurgicaux encore inoccupés. Même la fusion et l'hybridation d'une technique et/ou d'un instrument entre diverses spécialités peuvent représenter une invention nouvelle « par emprunt ».

Il faut également convenir que le contexte d'une innovation dans mon univers est probablement différent de ce qu'il est dans votre univers. Cela concerne non seulement les différences de contenu entre les spécialités chirurgicales, mais aussi l'influence des interactions sociales, environnementales et historiques particulières dont chacun de nous fait l'expérience. Il est aussi intéressant de tenir compte de l'état d'esprit particulier des chirurgiens qui recherchent l'innovation. Ces individus sont souvent motivés intérieurement, ils ont une grande force mentale ${ }^{3}$, savent encaisser les échecs sans perdre leur enthousiasme $e^{4}$ et croient que l'innovation fait partie de leur mission et de leur identité profondes de chirurgiens et/ou chirurgiens-chercheurs.

Même si ce n'est pas un critère absolu, les chirurgiens innovateurs peuvent bénéficier immensément d'environnements suralimentés; ce qui incluent des collègues solidaires, un soutien adéquat (tant financier que temporel) de la part des établissements/départements et un environnement physique propice à la collaboration, à la réflexion personnelle et à l'exécution des tâches. Dans nos hôpitaux, on oublie ce dernier aspect des milieux de travail, car chaque nouvelle recrue est reléguée à un bureau encombré en sous-sol, dans un bâtiment désorganisé et de plus en plus surpeuplé. On ne saurait surestimer l'importance de disposer d'environnements et de groupes pensés avec soin pour améliorer l'efficience et la productivité.

En plus d'identifier et d'étudier les individus créatifs, nous devons aussi nous demander comment mieux les 
soutenir'5. Il s'agit d'une démarche qui devient assurément de plus en plus difficile dans le monde actuel où les obstacles continuent de se multiplier dans les établissements universitaires et de santé publique provinciaux. À vrai dire, bon nombre de nos universités marquent le pas pour ce qui est d'identifier et de soutenir les individus qui ont un potentiel créatif et une feuille de route impeccable au plan de la productivité. Sans compter que plusieurs de nos systèmes de santé publics provinciaux sont confrontés à d'incessantes restrictions budgétaires et à des obligations en termes de volumes de patients; ce qui entre souvent en conflit avec les pratiques et les capacités en matière d'innovation. C'est peut-être pourquoi il est crucial que nos gouvernements provinciaux remettent à l'ordre du jour de généreux programmes de financement et de soutien à l'innovation, et des partenariats publics-privés qui iraient en ce sens ${ }^{1}$.

Malgré ces défis, les chirurgiens de diverses pratiques et de toutes les régions du pays doivent continuer d'encourager, de soutenir et de guider l'innovation dans leur pratique chirurgicale et celles de leurs collègues. Nos patients s'en porteront mieux, dès maintenant et à l'avenir.

\section{Chad G. Ball, MD, MSc; Edward J. Harvey, MD; Mohit Bhandari, MD}

Affiliations : Corédacteurs-en-chef, fournal canadien de chirurgie (Ball, Harvey); et Département de chirurgie, Université McMaster, Hamilton, Ont. (Bhandari).

Intérêts concurrents : E.J. Harvey est cofondateur et responsable de l'innovation médicale de NXTSens Inc.; cofondateur et médecinchef de MY01 Inc. et de Sensia Diagnostics Inc.; et cofondateur et directeur de Strathera Inc. Son établissement bénéficie du soutien de J et J DePuy Synthes, Stryker, MY01 et Zimmer. M. Bhandari déclare avoir reçu des subventions pour son établissement de la part des Instituts de recherche en santé du Canada, des National Institutes of Health, du Michael G. DeGroote Institute for Pain Research and Care et de la Physicians' Services Incorporated Foundation. Il déclare également des honoraires de consultation de Pendopharm, Bioventus et Acumed, et il est membre d'un comité consultatif du Mayo Clinic Core Center for Clinical Research. C.G. Ball ne déclare aucun intérêt concurrent.

Propriété intellectuelle du contenu : Il s'agit d'un article en libre accès distribué conformément aux modalités de la licence Creative Commons Attribution (CC BY-NC-ND 4.0), qui permet l'utilisation, la diffusion et la reproduction dans tout médium à la condition que la publication originale soit adéquatement citée, que l'utilisation se fasse à des fins non commerciales (c.-à-d., recherche ou éducation) et qu'aucune modifcation ni adaptation n'y soit apportée. Voir : https:// creativecommons.org/licenses/by-nc-nd/4.0/deed.fr.

Citation: Can J Surg 2021 November 18; 64(6). doi: 10.1503/ cjs. 019321

\section{Références}

1. Harvey E. Ed Harvey on innovation. Interviewed by Chad Ball and Ameer Farooq, Cold Steel: Canadian Fournal of Surgery Podcast; 2021 June 4. Available: https://soundcloud.com/cjs-podcast/ e85-ed-harvey-on-innovation (accessed 2021 Nov. 4).

2. Theil P. Zero to one: Notes on startups, or how to build the future. New York: Crown Business, Random House; 2014.

3. Percy DB, Streith L, Wong H, et al. Mental toughness in surgeons: Is there room for improvement? Can J Surg 2019;62:482-7.

4. Churchill WS. Churchill by bimself: in his own words. London: Rosetta Books; 2013.

5. Bhandari M. Mohit Bhandari on surgical trials, productivity and presentations. Interviewed by Chad Ball and Ameer Farooq, Cold Steel: Canadian Fournal of Surgery Podcast; 2020 June 12. Available: https://soundcloud.com/cjs-podcast/e20-mohit-bhandarion-surgical-trials-productivity-and-presentations (accessed 2021 Nov. 4). 